\title{
DYNAMIC BUCKLING BEHAVIOR OF MULTI-WALLED CARBON NANOTUBES SUBJECTED TO STEP AXIAL LOADING ${ }^{\star \star}$
}

\author{
Chengqi Sun ${ }^{1 \star} \quad$ Kaixin Liu ${ }^{2}$ Youshi Hong ${ }^{1}$ \\ $\left({ }^{1}\right.$ State Key Laboratory of Nonlinear Mechanics, Institute of Mechanics, Chinese Academy of Sciences, \\ Beijing 100190, China) \\ $\left({ }^{2}\right.$ LTCS and Department of Mechanics \& Aerospace Engineering, College of Engineering, Peking University, \\ Beijing 100871, China)
}

Received 10 December 2010, revision received 14 September 2011

\begin{abstract}
This paper studies the dynamic buckling behavior of multi-walled carbon nanotubes (MWNTs) subjected to step axial loading. A buckling condition is derived, and numerical results are presented for MWNTs under fixed boundary conditions. It is shown that the critical buckling load of MWNTs is of multi-branches and decreases as the time elongates. The associated buckling modes for different layers of MWNTs can be either in-phase or out of phase, which is related to the branch that the critical buckling load belongs to. For MWNTs with the same innermost tube radius, the critical buckling load is decreased when increasing the layers.
\end{abstract}

KEY WORDS multi-walled carbon nanotubes, dynamic buckling, van der Waals forces, continuum mechanics model

\section{INTRODUCTION}

Carbon nanotubes (CNTs) exhibit excellent mechanical properties and wide range of possible application $^{[1,2]}$. Many researchers have devoted to the study of mechanical behaviors for single-walled carbon nanotube (SWNT) or multi-walled carbon nanotubes (MWNTs) by using continuum mechanics model, molecular dynamic simulations and experiments ${ }^{[3-9]}$. For example, Yakobson et al. ${ }^{[10]}$ studied the axial buckling of SWNTs using molecular dynamics simulations and compared the results with a continuum shell model. Their results showed that the buckling behavior of an SWNT could be well predicted by a continuum shell model. $\mathrm{Ru}^{[11]}$ presented an elastic double-shell model and studied the axial compressive buckling of a double-walled carbon nanotube (DWNT). In Ru's analysis, an approximate linearized relation was adopted to model the van der Waals interaction between the outer and the inner nanotubes. Wang et al. ${ }^{[12]}$ studied the elastic buckling of MWNTs under high pressure based on a multiple-shell model and showed that the predicted critical pressure using continuum mechanics model was in reasonably good agreement with the experimental results by Tang et al. ${ }^{[13]}$. Wang et al. ${ }^{[14]}$ studied the size dependence of thin shell model for CNTs, and showed that the size dependence was insignificant for SWNTs with diameter larger than $1.5 \mathrm{~nm}$. While for SWNTs with diameter larger than $1.5 \mathrm{~nm}$, an isotropic thin shell model with constant thickness and elastic moduli could be used.

\footnotetext{
* Corresponding author. E-mail: scq@lnm.imech.ac.cn

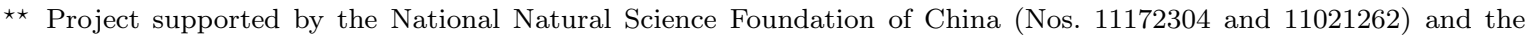
National Basic Research Program of China (No. 2012CB37500).
} 
Peng et al. ${ }^{[15]}$ studied the order of error by modeling SWNTs as thin shells via an atomistic-based finite-deformation shell theory from the point of view of structural response. In their paper, the ratio of atomic spacing $(\Delta \approx 0.14 \mathrm{~nm})$ to the radius of SWNT $\Delta / R$ was used to estimate the order of error. It was shown that, only for the order of error $O(\Delta / R)$ (as compared to unity), a universal constant shell thickness could be defined and an SWNT could be modeled as an elastic isotropic thin shell. Although CNTs are in nanoscale, many researches have shown that the continuum mechanics model can capture the main factor affecting the mechanical behavior of CNTs and can be an effective method for studying the mechanical behaviors of $\mathrm{CNTs}^{[16-20]}$.

As is shown in literature, most of previous studies have mainly focused on the static mechanical behaviors of MWNTs or MWNTs in various surroundings. CNTs can be used as basic elements of nanoscale devices, such as resonators, chemical and mechanical sensors ${ }^{[1,21,22]}$. Therefore, it is very essential to understand the dynamic mechanical property of MWNTs, which may be helpful for the potential application of CNTs. More recently, Sun and Liu ${ }^{[23]}$ studied the dynamic buckling behavior of DWNTs under step axial loading with the aid of stress wave propagation theory. It was indicated that the radii played an important role in dynamic buckling behavior of MWNTs. In this paper, a further study is performed for dynamic buckling behavior of MWNTs under step axial loading based on continuum mechanics model. Numerical results are worked out for the critical buckling load and the associated buckling mode of triple-walled CNTs under fixed boundary conditions. The effect of radii on the critical buckling load is also examined.

\subsection{Van der Waals Forces}

\section{MULTIPLE-SHELL MODEL}

Figure 1 shows the model of an MWNT under step axial loading. Subscripts $1,2, \cdots, N$ denote the corresponding quantities related to the outermost tube, its adjacent tube, ..., and the innermost tube, respectively. As used in literatures ${ }^{[11,17,18,23,24]}$, an approximate linearized relation is adopted to model the effect of van der Waals forces between adjacent tubes of MWNTs in this paper:

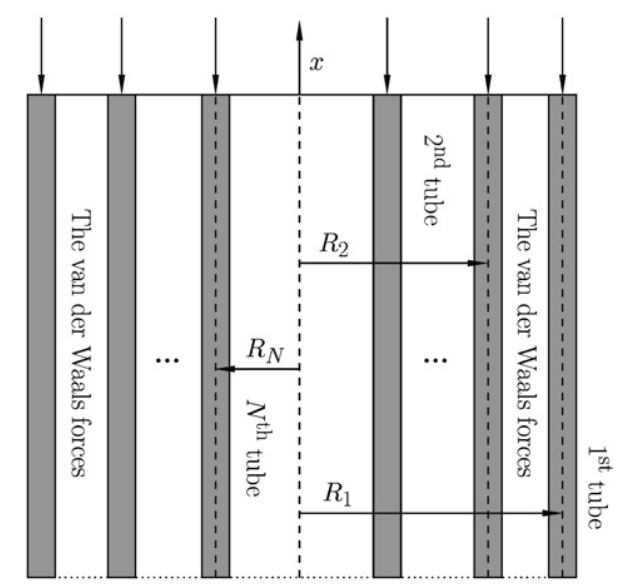

Fig. 1. A model of an MWNT under step axial loading.

$$
p_{k(k+1)}=c\left(w_{k+1}-w_{k}\right) \quad(k=1,2, \cdots, N-1)
$$

where $p_{k(k+1)}$ denotes the (inward) pressure (per unit area) exerted on the $k^{\text {th }}$ tube due to the $(k+1)^{\text {th }}$ tube, $w_{k}$ denotes the radial (inward) deflection of the $k^{\text {th }}$ tube due to buckling, and the van der Waals constant $c$ is estimated by

$$
c=\frac{320 \times \mathrm{erg} / \mathrm{cm}^{2}}{0.16 s^{2}} \quad \text { with } \quad s=1.42 \times 10^{-8} \quad \mathrm{~cm}
$$


Noting that the van der Waals interaction between the $k^{\text {th }}$ tube and the $(k+1)^{\text {th }}$ tube is equal and opposite, we have

$$
p_{(k+1) k}=-\frac{R_{k}}{R_{k+1}} p_{k(k+1)} \quad(k=1,2, \cdots, N-1)
$$

where $R_{k}$ denotes the radius of the $k^{\text {th }}$ tube.

\subsection{Buckling Equations}

In this paper, a perfect semi-infinite shell model impacted by a step axial load is considered, and the buckling is caused by the bifurcation of wave propagation. The study is based on the following assumptions $^{[23,25]}$. The axisymmetric buckling form occurs first and the buckling corrugations form at the initial stage. The longitudinal wave dominates and the effect of radial inertia can be negligible at the beginning of buckling.

For a perfect semi-infinite shell impacted by a step axial load $\sigma(t)=-\sigma_{x} H(t)$ at its end $(x=0)$, where $\sigma_{x}$ is a constant, $H(t)=1$ if $t \geq 0$, and $H(t)=0$ if $t<0$, the axial internal force satisfies

$$
N_{x}=\left\{\begin{array}{cc}
-\sigma_{x} h & \left(x \leq x_{e}\right) \\
0 & \left(x>x_{e}\right)
\end{array}\right.
$$

where $x_{e}=c_{e} t$, and $c_{e}=\left[E /\left(\rho-\rho \nu^{2}\right)\right]^{1 / 2}$ is the elastic wave speed.

According to Reissner's generalized variational principle and the theory of thin shells, the governing equation for a perfect thin shell with radial pressure can be expressed as ${ }^{[23,25]}$

$$
D \frac{\partial^{4} w}{\partial x^{4}}+\sigma_{x} h \frac{\partial^{2} w}{\partial x^{2}}+\frac{E h}{R^{2}} w+\rho h \frac{\partial^{2} w}{\partial t^{2}}-\frac{\rho h^{3}}{12} \frac{\partial^{4} w}{\partial x^{2} \partial t^{2}}-z=0 \quad\left(x \leq x_{e}\right)
$$

where $w(x, t)$ denotes the additional radial deflection measured from the initial perturbed deflection, and $z$ denotes the additional (inward) pressure due to buckling.

Now, the buckling problem on semi-infinite MWNTs is considered to be subjected to step axial loading. By supposing that each tube of an MWNT can be seen as a perfect elastic shell, the governing equations for dynamic buckling of an $N$-layer MWNT are obtained from Eqs.(1), (2), and (4) as

$$
\begin{aligned}
& D_{1} \frac{\partial^{4} w_{1}}{\partial x^{4}}+\sigma_{x} h_{1} \frac{\partial^{2} w_{1}}{\partial x^{2}}+\left(\frac{E_{1} h_{1}}{R_{1}^{2}}+c\right) w_{1}+\rho_{1} h_{1} \frac{\partial^{2} w_{1}}{\partial t^{2}}-\frac{\rho_{1} h_{1}^{3}}{12} \frac{\partial^{4} w_{1}}{\partial x^{2} \partial t^{2}}-c w_{2}=0 \quad\left(x \leq x_{e}\right) \\
& D_{k} \frac{\partial^{4} w_{k}}{\partial x^{4}}+\sigma_{x} h_{k} \frac{\partial^{2} w_{k}}{\partial x^{2}}+\left(\frac{E_{k} h_{k}}{R_{k}^{2}}+\frac{c R_{k-1}}{R_{k}}+c\right) w_{k} \\
&+\rho_{k} h_{k} \frac{\partial^{2} w_{k}}{\partial t^{2}}-\frac{\rho_{k} h_{k}^{3}}{12} \frac{\partial^{4} w_{k}}{\partial x^{2} \partial t^{2}}-\frac{c R_{k-1}}{R_{k}} w_{k-1}-c w_{k+1}=0 \quad\left(x \leq x_{e}\right) \\
& D_{N} \frac{\partial^{4} w_{N}}{\partial x^{4}}+\sigma_{x} h_{N} \frac{\partial^{2} w_{N}}{\partial x^{2}}+\left(\frac{E_{N} h_{N}}{R_{N}^{2}}+\frac{c R_{N-1}}{R_{N}}\right) w_{N} \\
&+\rho_{N} h_{N} \frac{\partial^{2} w_{N}}{\partial t^{2}}-\frac{\rho_{N} h_{N}^{3}}{12} \frac{\partial^{4} w_{N}}{\partial x^{2} \partial t^{2}}-\frac{c R_{N-1}}{R_{N}} w_{N}-1=0 \quad\left(x \leq x_{e}\right)
\end{aligned}
$$

where $D_{k}(k=1,2, \cdots, N)$ denotes the effective bending stiffness, $E_{k}$ denotes the Young's modulus, $\rho_{k}$ denotes the density and $h_{k}(k=1,2, \cdots, N)$ denotes the thickness of the $k^{\text {th }}$ tube.

Here, we take $D_{k}=D=0.85 \mathrm{eV}, E_{k} h_{k}=E h=360 \mathrm{~J} / \mathrm{m}^{2}$, and $\rho_{k} h_{k}=\rho h=\left(2.27 \mathrm{~g} / \mathrm{cm}^{3}\right) \times 0.34 \mathrm{~nm}$ $(k=1,2, \cdots, N)^{[10,17,18,23,24]}$.

\subsection{Buckling Condition}

\section{SOLUTION AND DISCUSSION}

By considering that the effect of radial inertia forces is very small before and at the beginning of the buckling occurrence, the radial inertia forces are neglected in the analysis for determining the buckling 
condition. Hence, Eq.(5) leads to

$$
\begin{aligned}
& D \frac{\partial^{4} w_{1}}{\partial x^{4}}+\tilde{N} \frac{\partial^{2} w_{1}}{\partial x^{2}}+\left(\frac{E h}{R_{1}^{2}}+c\right) w_{1}-c w_{2}=0 \quad\left(x \leq x_{e}\right) \\
& D \frac{\partial^{4} w_{k}}{\partial x^{4}}+\tilde{N} \frac{\partial^{2} w_{k}}{\partial x^{2}}+\left(\frac{E h}{R_{k}^{2}}+\frac{c R_{k-1}}{R_{k}}+c\right) w_{k}-\frac{c R_{k-1}}{R_{k}} w_{k-1}-c w_{k+1}=0 \quad\left(x \leq x_{e}\right) \\
& D \frac{\partial^{4} w_{N}}{\partial x^{4}}+\tilde{N} \frac{\partial^{2} w_{N}}{\partial x^{2}}+\left(\frac{E h}{R_{N}^{2}}+\frac{c R_{N-1}}{R_{N}}\right) w_{N}-\frac{c R_{N-1}}{R_{N}} w_{N-1}=0 \quad\left(x \leq x_{e}\right)
\end{aligned}
$$

where $\tilde{N}=\sigma_{x} h$.

The general solution of Eq.(6) can be expressed as

$$
w_{k}=U_{k} \mathrm{e}^{\lambda x} \quad(k=1,2, \cdots, N)
$$

where $U_{k}(k=1,2, \cdots, N)$ and $\lambda$ are constants.

Substitution of Eq.(7) into Eq.(6), a set of $N$ homogeneous equations for $U_{1}, U_{2}, \cdots, U_{N}$ are obtained as

$$
\begin{aligned}
& a_{11} U_{1}+a_{12} U_{2}=0 \\
& a_{k(k-1)} U_{k-1}+a_{k k} U_{k}+a_{k(k+1)} U_{k+1}=0 \quad(k=2,3, \cdots, N-1) \\
& a_{N(N-1)} U_{N-1}+a_{N N} U_{N}=0
\end{aligned}
$$

where

$$
\begin{aligned}
& a_{11}=D \lambda^{4}+\tilde{N} \lambda^{2}+c+\frac{E h}{R_{1}^{2}} \\
& a_{12}=-c \\
& a_{k(k-1)}=-c \frac{R_{k-1}}{R_{k}} \\
& a_{k k}=D \lambda^{4}+\tilde{N} \lambda^{2}+c \frac{R_{k-1}}{R_{k}}+c+\frac{E h}{R_{k}^{2}} \\
& a_{k(k+1)}=-c \\
& a_{N(N-1)}=-c \frac{R_{N-1}}{R_{N}} \\
& a_{N N}=D \lambda^{4}+\tilde{N} \lambda^{2}+c \frac{R_{N-1}}{R_{N}}+\frac{E h}{R_{N}^{2}}
\end{aligned}
$$

The requirement that Eq.(8) has non-zero solutions for $U_{k}(k=1,2, \cdots, N)$ leads to

$$
\operatorname{det} \boldsymbol{A}=0
$$

where $\boldsymbol{A}$ denotes the coefficient matrix on $U_{1}, U_{2}, \cdots, U_{N}$.

For an $N$-layer MWNT, $4 \times N$ eigenvalues $\lambda_{j}(j=1,2, \cdots, 4 \times N)$ on the axial load $\tilde{N}$ are obtained from Eq.(9). And the relation for $U_{1}, U_{2}, \cdots, U_{N}$ is obtained for a given $\lambda_{j}$, for example $U_{k}=U_{k}^{j} U_{1}$ $(k=2,3, \cdots, N)$. Thus, the buckling deflections are solved as

$$
w_{1}=\sum_{j=1}^{4 \times N} W_{j} \mathrm{e}^{\lambda_{j} x}, \quad w_{k}=\sum_{j=1}^{4 \times N} U_{k}^{j} W_{j} \mathrm{e}^{\lambda_{j} x} \quad(k=2,3, \cdots, N)
$$

where $W_{j}(j=1,2, \cdots, 4 \times N)$ is constant.

In this paper, the fixed boundary conditions are considered:

$$
w_{k}(0, t)=0, \quad \frac{\partial w_{k}}{\partial x}(0, t)=0 \quad(k=1,2, \cdots, N)
$$


Since the deflections and internal forces are zero in the non-disturbed region, the continuous conditions should be satisfied at the elastic wave front $x=x_{e}$, i.e.

$$
w_{k}\left(x_{e}, t\right)=0, \quad \frac{\partial w_{k}}{\partial x}\left(x_{e}, t\right)=0 \quad(k=1,2, \cdots, N)
$$

By substituting the solutions for the buckling deflections into Eqs.(11) and (12), $4 \times N$ equations are obtained on $W_{1}, W_{2}, \cdots, W_{4 N}$ :

$$
M \cdot Q=0
$$

where $\boldsymbol{M}$ denotes the coefficient matrix on $W_{1}, W_{2}, \cdots, W_{4 \times N}$ and $\boldsymbol{Q}=\left\{W_{1}, W_{2}, \cdots, W_{4 \times N}\right\}^{\mathrm{T}}$.

The requirement of Eq.(13) containing non-zero solutions provides the buckling condition, i.e.

$$
\operatorname{det} M=0
$$

By using Eq.(14), for a given time $t$ that buckling occurs, the critical buckling load is obtained, and then the associated buckling mode is determined from Eq.(10).

\subsection{Effect of Radial Inertia Forces}

During buckling, the effect of radial inertia forces begins to dominate and cannot be neglected. In this case, Eq. (5) has to be investigated. Let $w_{k}=B_{k} G(x, t)=B_{k} F(x) \mathrm{e}^{\xi t}$ (where $B_{k}$ is constant, and $\xi$ is a complex number), and take the Fourier sine transform of $w_{k}$ on $x$, then we have

$$
w_{k}=B_{k} \int_{0}^{\infty} g(\eta) \mathrm{e}^{\xi t} \sin (x \eta) \mathrm{d} \eta \quad(k=1,2, \cdots, N)
$$

where $g(\eta)=1 / \pi \int_{-\infty}^{+\infty} F(\zeta) \sin (\zeta \eta) \mathrm{d} \zeta$.

Substitution of Eq.(15) into Eq.(5) leads to

$$
\begin{aligned}
& b_{11} B_{1}+b_{12} B_{2}=0 \\
& b_{k(k-1)} B_{k-1}+b_{k k} B_{k}+b_{k(k+1)} B_{k+1}=0 \quad(k=2,3, \cdots, N-1) \\
& b_{N(N-1)} B_{N-1}+b_{N N} B_{N}=0
\end{aligned}
$$

where

$$
\begin{aligned}
& b_{11}=D \eta^{4}-\tilde{N} \eta^{2}+\rho h \xi^{2}+\frac{\rho h^{3} \eta^{2} \xi^{2}}{12}+c+\frac{E h}{R_{1}^{2}} \\
& b_{12}=-c \\
& b_{k(k-1)}=-c \frac{R_{k-1}}{R_{k}} \\
& b_{k k}=D \eta^{4}-\tilde{N} \eta^{2}+\rho h \xi^{2}+\frac{\rho h^{3} \eta^{2} \xi^{2}}{12}+c \frac{R_{k-1}}{R_{k}}+c+\frac{E h}{R_{k}^{2}} \\
& b_{k(k+1)}=-c \\
& b_{N(N-1)}=-c \frac{R_{N-1}}{R_{N}} \\
& b_{N N}=D \eta^{4}-\tilde{N} \eta^{2}+\rho h \xi^{2}+\frac{\rho h^{3} \eta^{2} \xi^{2}}{12}+c \frac{R_{N-1}}{R_{N}}+\frac{E h}{R_{N}^{2}}
\end{aligned}
$$

The requirement of Eq.(16) containing non-zero solutions on $B_{k}(k=1,2, \cdots, N)$ leads to

$$
\operatorname{det} \boldsymbol{B}=0
$$

where $\boldsymbol{B}$ denotes the coefficient matrix on $B_{1}, B_{2}, \ldots, B_{N}$.

From Eq.(17), we have

$$
\xi^{2}=\frac{-D \eta^{4}+\tilde{N} \eta^{2}+X_{i}}{\rho h+\rho h^{3} \eta^{2} / 12}
$$


where $X_{1}, X_{2}, \ldots, X_{N}$ denote the roots of Eq.(17) on $D \eta^{4}-\tilde{N} \eta^{2}+\left(\rho h+\rho h^{3} \eta^{2} / 12\right) \xi^{2}$.

Now, see the roots of $\xi^{2}=\frac{-D \eta^{4}+\tilde{N} \eta^{2}+X_{1}}{\rho h+\rho h^{3} \eta^{2} / 12}$ on $\xi$ first.

Case $\left(\right.$ a): $\tilde{N}^{2}+4 D X_{1}>0$. Equation $-D \eta^{4}+\tilde{N} \eta^{2}+X_{1}=0$ has two distinct real roots $\eta_{1}^{2}=$ $\left(\tilde{N}-\sqrt{\tilde{N}^{2}+4 D X_{1}}\right) /(2 D)$ and $\eta_{2}^{2}=\left(\tilde{N}+\sqrt{\tilde{N}^{2}+4 D X_{1}}\right) /(2 D)$ on $\eta^{2}$. When $\eta_{1}^{2}<\eta^{2}<\eta_{2}^{2}, \xi$ takes a positive root, and $G(x, t)$ increases with $t$. Thus, the MWNT may buckle. And the associated buckling wave length $L$ satisfies $2 \pi / \eta_{2}<L<2 \pi / \eta_{1}$, i.e., the buckling wave length will be neither too long nor too short. When $\eta^{2}=\eta_{1}^{2}$ or $\eta^{2}=\eta_{2}^{2}, \xi^{2}=0$, the solution $G(x, t)$ is described as $\left(a_{0}+a_{1} t\right) F(x)$ (where $a_{0}$ and $a_{1}$ are constants), and the buckling may occur. When $\eta^{2}<\eta_{1}^{2}$ or $\eta^{2}>\eta_{2}^{2}$, equation $\xi^{2}=\frac{-D \eta^{4}+\tilde{N} \eta^{2}+X_{1}}{\rho h+\rho h^{3} \eta^{2} / 12}$ has only pure imaginary roots on $\xi$. Noting that $\xi= \pm \xi_{0} \mathrm{i}$ (where $\xi_{0}$ is real number, and $\left.\xi_{0}>0\right)$, the solution $G(x, t)$ can be expressed as the linear combination of $\sin \left(x \eta \pm \xi_{0} t\right)$ and $\cos \left(x \eta \pm \xi_{0} t\right)$, which represents the form of vibration but not buckling.

Case (b): $\tilde{N}^{2}+4 D X_{1}=0$. Equation $-D \eta^{4}+\tilde{N} \eta^{2}+X_{1}=0$ has double root $\eta^{2}=\tilde{N} /(2 D)$ on $\eta^{2}$. Similar to the analysis in Case (a), the buckling can only occur when $\eta^{2}=\tilde{N} /(2 D)$. The critical buckling load determined by $\tilde{N}^{2}+4 D X_{1}=0$ represents the corresponding one for static buckling.

Case (c): $\tilde{N}^{2}+4 D X_{1}<0$. In this case, $-D \eta^{4}+\tilde{N} \eta^{2}+X_{1}<0$. Equation $-D \eta^{4}+\tilde{N} \eta^{2}+X_{1}=0$ has only pure imaginary roots on $\xi$. Hence, the buckling cannot take place.

Completely analogous analysis can be used to deal with the roots of $\xi^{2}=\frac{-D \eta^{4}+\tilde{N} \eta^{2}+X_{i}}{\rho h+\rho h^{3} \eta^{2} / 12}(i=2$, $3, \cdots, N)$ on $\xi$, and similar results are obtained.

\subsection{Buckling Condition}

In the following, numerical results are worked out for MWNTs with dimensionless number $T=$ $c_{e} t / h$.

Figure 2 shows the variation of critical buckling load versus $T$ for a triple-walled CNT with innermost tube radius $R_{3}=2 \mathrm{~nm}$. It is seen that the critical buckling load versus $T$ is of multi-branches. We call them the first branch, the second branch and so on from the lowest one. Figure 2 indicates that the critical buckling load decreases as the time elongates, and the higher critical buckling load corresponds to the shorter time buckling occurs for each branch. This result is similar to that of the Fig. 2 Variation of critical buckling load versus $T$ for a triplecritical buckling load for a DWNT versus the di- walled CNT with innermost tube radius $R_{3}=2 \mathrm{~nm}$. mensionless number $T=c_{e} t / h$ given by Sun and Liu ${ }^{[23]}$.

Figure 3 shows sketches of some buckling modes associated with the first, second and third branch of critical buckling load for a triple-walled CNT with innermost tube radius $R_{3}=2 \mathrm{~nm}$. It is seen from Fig. 3 that the associated buckling modes for different layers of the triple-walled CNT can be either in-phase (see Figs.3(a)-3(c)) or out of phase (see Figs.3(d) and 3(e)), which is related to the branch that the critical buckling load belongs to. This phenomenon can also be found for dynamic buckling behavior of DWNTs under step axial loading ${ }^{[23]}$ indicating that, for dynamic buckling behavior of MWNTs, the associated buckling form is not only dependent on the magnitude of the critical buckling load, but also on the branch the critical buckling load belongs to. Figure 3 also indicates that, for the first branch of critical buckling load (see Figs.3(a)-3(c)), the buckling wave length increases gradually with the increase of time. Once the buckling wave length reaches to a certain value, the buckling wave number begins to increase. This result is consistent with the analysis that the buckling wave length is neither too long nor too short in $§ 3.2$. In addition, a comparison of the buckling form with that for DWNTs ${ }^{[23]}$ shows that, there is one form for the out-of-phase mode of DWNTs and two forms for the out-of-phase modes of triple-walled CNTs (see Figs.3(d) and 3(e)), indicating that the variety of out-of-phase modes increases with increasing the layers of MWNTs. 


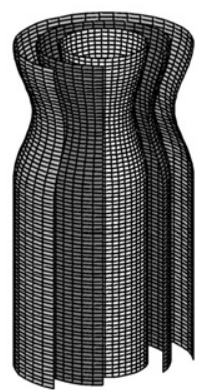

(a)

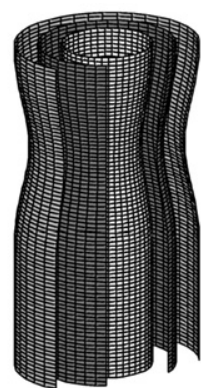

(b)

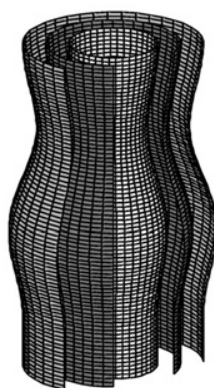

(c)

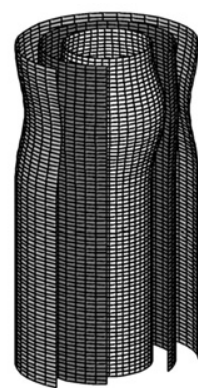

(d)

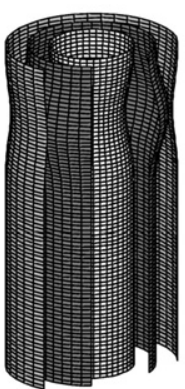

(e)

Fig. 3. Sketches of some buckling modes associated with the first, second and third branch of critical buckling load for a triple-walled CNT with innermost tube radius $R_{3}=2 \mathrm{~nm}$. (a) First branch at $T=2$, (b) First branch at $T=3$, (c) First branch at $T=4$, (d) Second branch at $T=2$, (e) Third branch at $T=2$.

Figure 4 shows the variation of the first, second and third branch of critical buckling load versus innermost tube radius at $T=11$ for triple-walled CNTs. It is observed that the first three branches of critical buckling load have very similar variation trends with regard to innermost tube radius. They all decrease with increasing innermost tube radius, especially for smaller innermost tube radius.

Figure 5 plots the static and first branch of critical buckling load for DWNTs, triple-walled CNTs and quadruple-walled CNTs with the same innermost tube radius at $T=11$, showing that, for MWNTs with the same innermost tube radius, the Fig. 4 Variation of the first, second and third branch of critcritical buckling load is decreased when increasing ical buckling load with innermost tube radius at $T=11$ for the layers of MWNTs. Moreover, the variation of triple-walled CNTs.

critical buckling load versus the layers of MWNTs is related to the radii of MWNTs. For MWNTs with innermost tube radius about several nanometers, the variation of critical buckling load versus the layers of MWNTs is obvious. While for MWNTs with large innermost tube radius, the critical buckling load is almost independent of the variation of the layers of MWNTs.

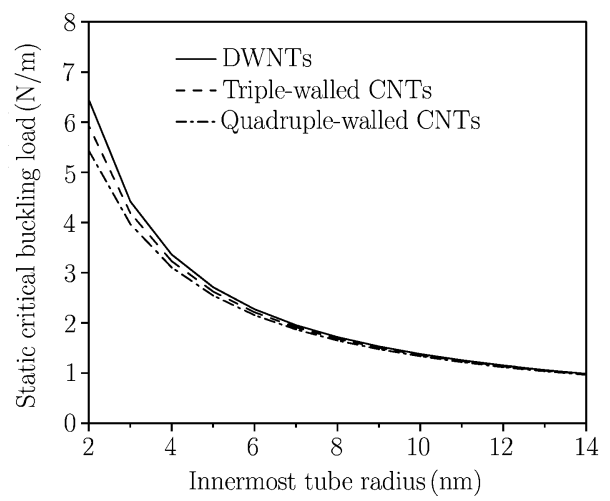

(a)

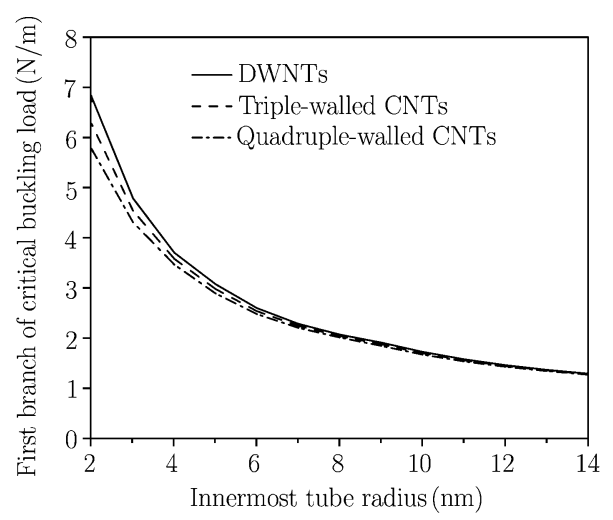

(b)

Fig. 5. Static and first branch of critical buckling load for DWNTs, triple-walled CNTs and quadruple-walled CNTs with the same innermost tube radius at $T=11$. 


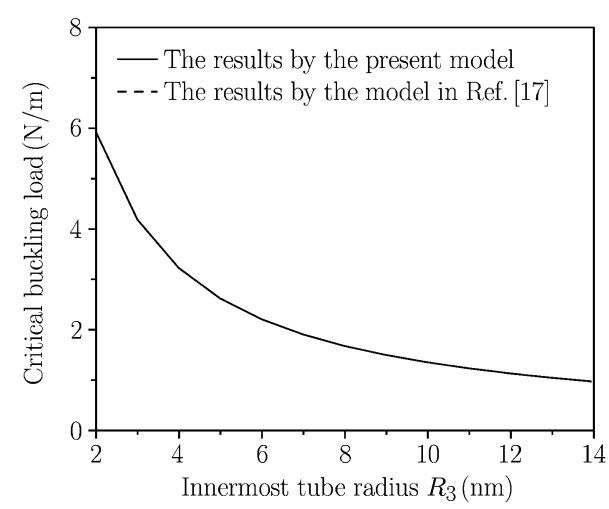

Fig. 6. Static critical buckling load of triple-walled CNTs by the presented model with comparison to the one obtained by the model in Ref.[17] for axisymmetric compressive buckling at length-to-outermost tube radius ratio $l / R_{1}=12$.

Figure 6 shows the result of the static critical buckling load of triple-walled CNTs by the presented model with comparison to the one obtained by the model in Ref.[17] for axisymmetric compressive buckling at length-to-outermost tube radius ratio $l / R_{1}=12$. It is seen that the static critical buckling load by the presented model is in well agreement with the one by the model in literature (the relative variation is less than $0.02 \%$ with the scale of the innermost tube radius ranging from $2 \mathrm{~nm}$ to $14 \mathrm{~nm}$ ), which indicates the validity of the present model.

\section{CONCLUSIONS}

With the aid of stress wave propagation theory, a study is performed for dynamic buckling behavior of MWNTs under step axial loading based on continuum mechanics model, which takes into account the van der Waals forces between adjacent layers. A buckling condition is derived, and the effect of radial inertia forces is discussed. Numerical results are illustrated for dynamic buckling behavior of MWNTs under fixed boundary conditions. It is indicated that the critical buckling load of MWNTs is of multi-branches and decreases as time variable elongates. The associated buckling modes for different layers of MWNTs can be either in-phase or out of phase, which is related to the branch the critical buckling load belongs to. Moreover, the variety of out-of-phase modes increases with the increase of the layers of MWNTs. Additionally, the static critical buckling load of triple-walled CNTs by the presented model is compared with the one by the model in literature, which shows well agreement. It is noted that, the MWNTs are actually in finite length, and the reflection of stress wave would have influences on the buckling behavior of MWNTs. The present model has its approximation on modeling each tube of MWNTs as a perfect semi-infinite elastic shell.

\section{References}

[1] Ajayan,P.M. and Zhou,O.Z., Applications of carbon nanotubes. Topics in Applied Physics, 2001, 80: 391425.

[2] Qian,D., Wagner,G.J., Liu,W.K., Yu,M.F. and Ruoff,R.S., Mechanics of carbon nanotubes. Applied mechanics reviews, 2002, 55: 495-533.

[3] Zhang,Y.Q., Liu,G.R. and Wang,J.S., Small-scale effects on buckling of multiwalled carbon nanotubes under axial compression. Physical review B, 2004, 70: 205430-1-205430-6.

[4] Chang,T.C., Guo,W.L. and Guo,X.M., Buckling of multiwalled carbon nanotubes under axial compression and bending via a molecular mechanics model. Physical review B, 2005, 72: 064101-1-064101-11.

[5] Waters,J.F., Guduru,P.R., Jouzi,M. and Xu,J.M., Shell buckling of individual multiwalled carbon nanotubes using nanoindentation. Applied Physics Letters, 2005, 87: 103109-1-103109-3.

[6] Huang,Y., Wu,J. and Hwang,K.C., Thickness of graphene and single-wall carbon nanotubes. Physical review $B, 2006,74:$ 245413-1-245413-9.

[7] Fu,Y., Bi,R. and Zhang,P., Nonlinear dynamic instability of double-walled carbon nanotubes under periodic excitation. Acta Mechanica Solida Sinica, 2009, 22(3): 206-212.

[8] Lu,W.B. and Wu,J., A continuum model for axial-strain-induced torsion in single-wall carbon nanotubes. Acta Mechanica Solida Sinica, 2009, 22(4): 283-286. 
[9] Sun,C. and Liu,K., Combined torsional buckling of multi-walled carbon nanotubes coupling with axial loading and radial pressures. International Journal of Solids and Structures, 2008, 45: 2128-2139.

[10] Yakobson,B.I., Brabec,C.J. and Bernholc,J., Nanomechanics of carbon tubes: instability beyond linear response. Physical Review Letters, 1996, 76: 2511-2514.

[11] Ru,C.Q., Effect of van der Waals forces on axial buckling of a double-walled carbon nanotube. Journal of Applied Physics, 2000, 87: 7227-7231.

[12] Wang,C.Y., Ru,C.Q. and Mioduchowski,A., Elastic buckling of multiwall carbon nanotubes under high pressure. Journal of Nanoscience and Nanotechnology, 2003, 3: 199-208.

[13] Tang,D.S., Bao,Z.X., Wang,L.J., Chen,L.C., Sun,L.F., Liu,Z.Q., Zhou,W.Y. and Xie,S.S., The electrical behavior of carbon nanotubes under high pressure. Journal of Physics and Chemistry of Solids, 2000, 61: 1175-1178.

[14] Wang,L.F., Zheng,Q.S., Liu,J.Z. and Jiang,Q., Size dependence of the thin-shell model for carbon nanotubes. Physical Review Letters, 2005, 95: 105501-1-105501-4.

[15] Peng,J., Wu,J., Hwang,K.C., Song,J. and Huang,Y., Can a single-wall carbon nanotube be modeled as a thin shell? Journal of the Mechanics and Physics of Solids, 2008, 56: 2213-2224.

[16] Harik,W.M., Ranges of applicability for the continuum-beam model in the constitutive analysis of carbonnanotubes and nanorods. Solid State Communications, 2001, 120: 331-335.

[17] Wang,C.Y., Ru,C.Q. and Mioduchowski,A., Axially compressed buckling of pressured multiwall carbon nanotubes. International Journal of Solids and Structures, 2003, 40: 3893-3911.

[18] Wang,C.Y., Ru,C.Q. and Mioduchowski,A., Applicability and limitations of simplified elastic shell equations for carbon nanotubes. ASME Journal of Applied Mechanics, 2004, 71: 622-631.

[19] Akita,S., Nishio,M. and Nakayama,Y., Buckling of multiwall carbon nanotubes under axial compression. Japanese Journal of Applied Physics, 2006, 45: 5586-5589.

[20] Sears,A. and Batra,R.C., Buckling of multiwalled carbon nanotubes under axial compression. Physical Review B, 2006, 73: 085410-1-085410-11.

[21] Dharap,P., Li,Z.L., Nagarajaiah,S. and Barrera,E.V., Nanotube film based on single-wall carbon nanotubes for strain sensing. Nanotechnology, 2004, 15: 379-382.

[22] Li,C.Y. and Chou,T.W., Strain and pressure sensing using single-walled carbon nanotubes. Nanotechnology, 2004, 15: 1493-1496.

[23] Sun,C. and Liu,K., Dynamic buckling of double-walled carbon nanotubes under step axial load. Acta Mechanica Solida Sinica, 2009, 22(1): 27-36.

[24] Wang,X., Lu,G. and Lu,Y.J., Buckling of embedded multi-walled carbon nanotubes under combined torsion and axial loading. International Journal of Solids and Structures, 2007, 44: 336-351.

[25] Xu,X.S., Su,X.Y. and Wang,R., Dynamic buckling of elastic-plastic cylindrical shells and axial stress waves. Science in China (Series A), 1995, 38: 472-480. 